Cf

the study reveals a key mechanistic role for the UPR

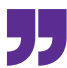

treatment in cell lines, suggesting that IRE1 $\alpha-X B P 1 s$ signalling also regulates MYC oncogene activity. This relationship was further corroborated in published data sets and a prostatectomy tissue microarray $(n=260)$, and additional chromatin immunoprecipitation (ChIP) and reporter assays illustrated that MYC is directly transcriptionally regulated by XBP1s.

In demonstration of the prognostic value of these findings, a five-gene XBP1 expression signature was shown to have clear predictive power for disease-free survival and biochemical-recurrence-free survival in three cohorts.

Overall, the study reveals a key mechanistic role for the UPR, via IRE1 $\alpha-\mathrm{XBP} 1 \mathrm{~s}$ and MYC signalling, in prostate cancer and, in doing so, identifies a potential drug candidate to target this prosurvival mechanism.

Conor A. Bradley

ORIGINAL ARTICLE Sheng, X. et al. IRE1 $\alpha-X B P 1 s$ pathway promotes prostate cancer by activating c-MYC signaling. Nat. Commun. 10, 323 (2019)

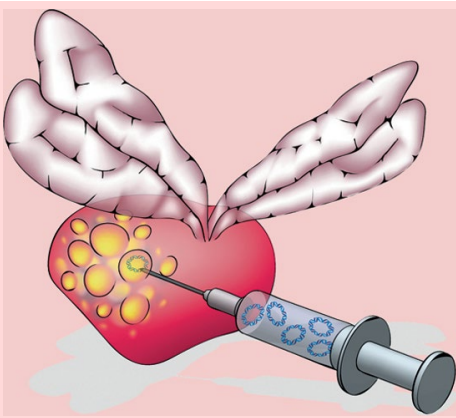

injection of PSAtk-VLPs followed by intraperitoneal injection of ganciclovir substantially inhibited tumour growth; however, this effect was not seen in a bladder cancer xenograft model. These results show that specific targeting of CRPC using gene therapy is possible and that delivery of the treatment through the circulatory system is feasible. Thus, this therapy could be an alternative future treatment option for men with this disease.

Louise Stone

ORIGINAL ARTICLE Lin, M.-C. et al. Gene therapy for castration-resistant prostate cancer cells using JC polyomavirus-like particles packaged with a PSA promoter driven-suicide gene. Cancer Gene Ther. https://doi.org/10.1038/ s41417-019-0083-0 (2019)
FROM THE MEETING

\section{ASCO Genitourinary Cancers Symposium}

In February 2019, 4000 international delegates attended the 2019 Genitourinary Cancers Symposium from the American Society of Clinical Oncology (ASCO GU) in San Francisco, which featured an array of multidisciplinary sessions spanning urologic oncology. The 2019 proceedings were opened by scientific chair Daniel Hamstra, who noted the gradual increase in attendance of approximately one-third since 2016 and the corresponding rise in the number of abstracts to $\sim 800$.

The first day kicked off with an excellent session on optimizing the management of clinically significant nonmetastatic prostate cancer, with a notable talk from Samir Taneja on the evolution and role of MRI in targeted biopsy. Taneja posed a crucial question to clinicians - "are you more comfortable missing a small number of high-grade cancers and monitoring those patients, or continuing to detect a large number of low-grade cancers?" The oral abstract session was highlighted by the first interim findings from the phase III ARAMIS trial, presented by Karim Fizazi, showing that darolutamide significantly improved metastasis-free survival compared with placebo in nonmetastatic castration-resistant prostate cancer (CRPC) and had a favourable safety profile that was comparable between arms.

On day two, the bar was set for the session on challenges and advances in perioperative and local therapy for urothelial carcinoma with a topical debate on the optimal local treatment for muscle-invasive bladder cancer - Michael Cookson argued for a surgical approach, whereas Jason Efstathiou advocated a trimodal therapy approach for bladder preservation. This discussion was wisely followed by a multidisciplinary case panel on multimodality treatment in challenging cases of urothelial carcinoma. James Gulley gave the well-attended keynote lecture on immunotherapy across genitourinary malignancies, during which he highlighted recent data and clinical challenges related to the distinct immunological, genomic and molecular landscapes of bladder, renal and prostate cancer.

The final day started with a session on evolving management of metastatic renal cell carcinoma (RCC), during which Samra Turajlic presented thoughtprovoking insights on RCC genetic evolution from the landmark TRACERx Renal study. The oral abstract session was dominated by large immune checkpoint inhibitor (ICl) trials, with Tom Powles presenting the widely anticipated data from the phase III KEYNOTE-426 study, showing the superior efficacy of pembrolizumab plus axitinib over sunitinib as first-line therapy for locally advanced or metastatic RCC. However, following the focus on immunotherapy for clear cell RCC (cCRCC) in previous years, new data were reported this year in non-ccRCC (nccRCC). Indeed, Rana McKay noted that the atezolizumab-bevacizumab combination had antitumour activity in a phase II study of nccRCC and ccRCC with sarcomatoid differentiation, and David McDermott presented exciting data from cohort B of the phase II KEYNOTE-427 trial, showing the promising activity of first-line pembrolizumab in advanced nccRCC.

Presentations from the meeting are available to watch on the symposium website. We eagerly await next year's ASCO GU symposium in San Francisco.

Conor A. Bradley

FURTHER READING TRACER $x$ Renal consortium. TRACERx Renal: tracking renal cancer evolution through therapy. Nat. Rev. Urol. 14, 575-576 (2017) | Powles, T. \& Morrison, L. Biomarker challenges for immune checkpoint inhibitors in urothelial carcinoma. Nat. Rev. Urol. 15, 585-587 (2018) 